\title{
Il coaching nel wound care. L'infermiere specialista in wound care, facilitatore nella gestione delle ferite cutanee tra ospedale e territorio nell'Azienda AUSL di Bologna: una proposta progettuale
}

\author{
Rossana Quatrini \\ Unità Assistenziale - Piastra Medica Ospedale San Giovanni in Persiceto, Azienda AUSL di Bologna, Bologna, Italia
}

\section{RIASSUNTO}

Questo progetto nasce con l'idea di elaborare una strategia organizzativa/professionale che possa favorire un'omogeneizzazione dei comportamenti dei professionisti sanitari, determinando una riduzione dei costi in termini di risparmio di risorse umane ed economiche, ma soprattutto favorire un miglioramento della qualità di vita dei soggetti portatori di lesioni cutanee. Le lesioni cutanee, ma soprattutto le ferite di difficile guarigione, si caratterizzano per una lunga durata e un'elevata incidenza di complicanze, che spesso si traducono in un considerevole onere economico. L'infermiere Specialista Wound Care, collaborando attivamente con i professionisti sanitari attraverso attività di consulenza, formazione on work, supervisione dei processi operativi, si pone come un'importante figura di riferimento in termini di miglioramento degli obiettivi di cura, nonché degli esiti di cura. Il progetto, di dimensioni Aziendali, si sviluppa in setting assistenziali specifici Ospedale-Territorio e lo Specialista Wound Care opera in collaborazione con gli infermieri di quei setting attraverso una metodologia di Coaching.

\section{INTRODUZIONE}

La difficoltà crescente della gestione delle ferite, acute e croniche, viene imputata soprattutto ad una difformità di erogazione di assistenza ai soggetti affetti da tali problematiche. L'OMS sostiene che è di fondamentale importanza la collaborazione interprofessionale, sia per quanto riguarda la formazione, che la pratica gestionale delle ferite al fine di fornire la cura migliore al paziente. Le Linee Guida internazionali suggeriscono che per prevenire e gestire le ferite, come le ulcere da pressione, con successo è necessario un approccio di squadra. ${ }^{1}$

Corrispondenza: Rossana Quatrini, Unità Assistenziale - Piastra Medica Ospedale San Giovanni in Persiceto, Azienda AUSL di Bologna, Bologna, Italia.

E-mail: rossanaquattrini@libero.it

Key words: Cura delle ferite; Coaching; Infermieristica; Lesioni cutanee.

Conflitto d'interesse: l'autore dichiara l'assenza di conflitti d'interesse.

Fondi: nessuno.

Ricevuto per la pubblicazione: 30 maggio 2018 .

Accettato per la pubblicazione: 4 febbraio 2019.

This work is licensed under a Creative Commons Attribution NonCommercial 4.0 License (CC BY-NC 4.0).

CC Copyright R. Quatrini, 2019

Licensee PAGEPress, Italy

Italian Journal of Wound Care 2019; 3(1):32-37

doi:10.4081/ijwc.2019.37
L'Azienda AUSL di Bologna, una delle più grandi e rilevanti aziende socio-sanitarie del Paese con oltre 1,7 miliardi di euro di bilancio annuo e più di 8.000 dipendenti, ha sviluppato una grande sensibilità rispetto al problema lesioni cutanee: consta di 6 Distretti di committenza e garanzia, l'assistenza distrettuale viene garantita attraverso strutture gestite direttamente dall'Azienda AUSL e dal Privato convenzionato. La rete ospedaliera, pubblica e privata accreditata, ha una presenza capillare sul territorio e garantisce l'autosufficienza aziendale non solo sulle discipline di base, ma anche su quelle di più elevato livello specialistico.

Il Servizio Infermieristico Domiciliare è collegato ai sei Distretti e fornisce assistenza agli utenti attraverso 24 punti di erogazione dislocati per coprire l'intero bacino di utenza territoriale aziendale; il personale infermieristico si occupa anche degli ambulatori infermieristici territoriali, delle Case della Salute e degli stabilimenti ospedalieri che fanno capo al territorio di riferimento. ${ }^{2}$

Da un'analisi recente sulle prestazioni per le medicazioni si evince quanto segue.

Per le Degenze Ospedaliere non è possibile quantizzare le prestazioni di medicazione o il numero di pazienti con lesioni cutanee in quanto la documentazione assistenziale è solo cartacea e nelle schede di dimissione ospedaliera non è quasi mai compilato il DRG (diagnosis-related group) corrispondente, è però strutturato un programma di monitoraggio dell'incidenza e della prevalenza delle lesioni da pressione solo nelle aree mediche aziendali. Per gli Ambulatori Infermieristici Territoriali il numero totale di prestazioni di medicazioni/bendaggio sono circa 60.000/anno. Per l'Assistenza Domiciliare il numero totale di prestazioni di medicazioni/bendaggio sono circa 200.000/anno. 
L'Azienda AUSL di Bologna sta consolidando lo sviluppo di percorsi di cura che offrano a ogni cittadino la garanzia di accedere a cure tempestive e adeguate per expertise professionale individuale, di equipe e di struttura (service mix) - definibili come percorsi diagnostico-terapeutico-assistenziali; il Percorso Diagnostico Terapeutico Assistenziale del paziente con ferite difficili è tra questi.

Il progetto del Percorso Diagnostico Terapeutico Assistenziale delle Ferite Difficili(PDTA) delle ferite difficili ha voluto superare l'assenza di una rete uniforme che accompagna il paziente con ferita difficile nel suo percorso per problematicità di interazione fra i medici di medicina generale (MMG), gli specialisti e gli infermieri, ma anche dalla non omogeneità di approcci alle lesioni cutanee nella fase di prevenzione e a trattamenti spesso inadeguati della ferita acuta che si trasforma in cronica allungando così in modo considerevole i tempi di guarigione e aumentando in modo esponenziale i costi. L'azione principale è stata quella di intercettare il paziente con lesioni cutanee, trattarlo in modo adeguato e indirizzarlo nel posto giusto qualora la lesione cutanea non dia segni di guarigione in tempi ragionevoli: entro 8-10 settimane dalla comparsa, dopo tale termine la lesione diventa cronica. ${ }^{3}$

L'Azienda AUSL di Bologna ha nel suo organico delle risorse professionali che si occupano da tempo della gestione delle ferite cutanee nei diversi setting assistenziali, un gruppo di Infermieri con background formativo ed esperienziale attestante la competenza specifica, molti con master in Wound Care, che svolgono il ruolo da referenti/Coach. I referenti Coach fanno parte, inoltre, di una rete corposa di infermieri che, attraverso una partecipazione attiva, collaborano a progetti aziendali quali poster illustrati, procedure operative ed informative come ad es: Guida alla scelta della medicazione, ${ }^{4}$ audit documentali, visite ispezionali (educational outretch visit) per la raccolta dati ai fini di monitorare la prevalenza e l'incidenza delle lesioni cutanee.

Il Coach viene visto, quindi, come un facilitatore nei processi di cambiamento e di sviluppo di individui e di organizzazioni, il mezzo di trasporto da una situazione di partenza alla meta desiderata.

Nonostante vi siano stati forniti gli strumenti operativi (PDTA ferite difficili, sistemi operativi informatizzati, ambulatori specialistici, ecc.) e la formazione rivesta un ruolo fondamentale nella partecipazione collettiva per la crescita professionale nonché delle competenze specifiche, da recenti audit, condotti in aree territoriali e degenze ospedaliere dell'Azienda si evince ancora una notevole difformità nell'utilizzo di best practice proprio nel rispetto della prevenzione, della valutazione e del trattamento delle lesioni cutanee. Si avverte la necessità di costruire delle relazioni, delle collaborazioni tra infermieri specialisti, esperti e competenti, e colleghi che si occupano di lesioni, accompagnandoli nel cambiamento dei comportamenti non sempre adeguati (Figura 1).

\section{Obiettivo}

Il presupposto fondamentale su cui si basa questo progetto è legato al fatto che tutti gli operatori professionali, che operano nella gestione delle ferite cutanee sia in ospedale sia sul territorio, partecipano alla formazione per la prevenzione, la valutazione e il trattamento delle lesioni da pressione, valutazione e trattamento delle lesioni vascolari, con specifiche rispetto alle lesioni di diversa eziologia, basata su evidenze scientifiche dettate da Linee Guida internazionali e Protocollo Regionale emanato dalla Regione Emilia Romagna, ${ }^{5,6}$ con lo scopo di omogeneizzare i comportamenti clinico-assistenziali. Durante gli eventi formativi vengono trasferiti concetti legati all'assessment delle ferite cutanee, la loro valutazione secondo scale validate, il loro trattamento secondo l'utilizzo di medicazioni avanzate, la loro registrazione attraverso strumenti operativi cartacei e informatici generando un miglioramento in termini di concetti teorici ma ancora distanti dalla realtà operativa di natura pratica.

Presupposto secondario, ma non meno importante, è l'utilizzo della metodologia di Coaching per le strategie relazionali del Facilitatore Specialista Wound Care, favorenti una crescita motivazionale dei professionisti coinvolti.

L'obiettivo generale è quello di creare una forma di integrazione tra ospedale e territorio per la gestione della prevenzione e della cura delle lesioni cutanee attraverso l'inserimento di un infermiere Specialista/Esperto/Competente Wound Care che attraverso il ruolo di facilitatore, riesca a collaborare in maniera attiva e costruttiva a fianco degli operatori professionali, agevolando così le decisioni professionali, superando la frammentazione degli interventi ancora in essere, favorendo una crescita delle competenze ai fini del raggiungimento delle best practices, attraverso una metodologia di Coaching.

L'obiettivo specifico è quello di favorire la prevenzione delle lesioni da pressione a domicilio sui pazienti fragili; di favorire la supervisione delle lesioni cutanee complesse nell'ambito ospedaliero e territoriale attraverso la fornitura di consulenze specialistiche infermieristiche; di favorire la creazione di un collegamento dei vari setting Territoriali e Ospedalieri per superare la frammentazione degli interventi.

\section{MATERIALI E METODI}

Il Responsabile Area Nord Direzione Assistenziale Tecnica Riabilitativa (DATeR), dopo aver consultato il Responsabile Assistenziale del PDTA delle ferite difficili sulla fattibilità del progetto in oggetto, coinvolge il Responsabile DATeR Unità Operativa di San Giovanni in 
Persiceto Pianura Ovest per la partecipazione attiva nel progetto: da qui il mandato per la stesura del progetto.

Il progetto coinvolgerà lo stabilimento ospedaliero di San Giovanni in Persiceto, i servizi infermieristici domiciliari e ambulatoriali del Nucleo di Cure Primarie nel Distretto Pianura Ovest, MMG del territorio interessato, il Punto di Coordinamento di Assistenza Primaria (PCAP) Pianura Ovest.

Verrà costruita una modalità di coinvolgimento e di interazione tra l'infermiere Specialista facilitatore e gli infermieri dei diversi setting assistenziali: l'infermiere Specialista Wound Care dovrà esprimere autorevolezza nelle fasi di integrazione e di collaborazione con gli operatori sanitari; dovrà mantenere un'intesa efficace e costruttiva con la rete del PDTA delle ferite difficili; dovrà collaborare con la Dirigenza Infermieristica per la risoluzione di eventuali problematiche legate alla gestione di lesioni cutanee nei diversi setting assistenziali.

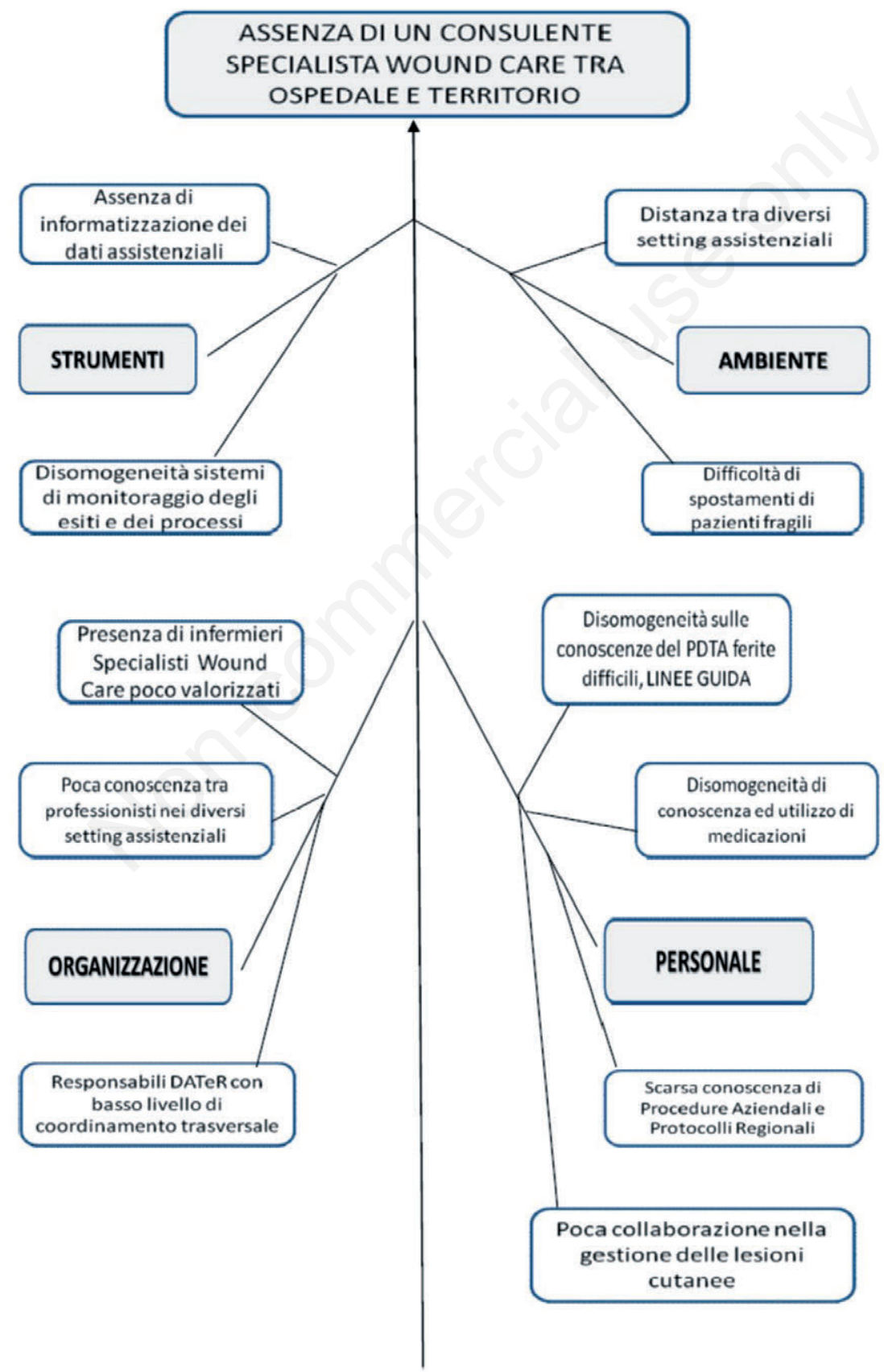

Figura 1. Diagramma di Ishikawa che mostra la mancanza di un consulente per la cura delle ferite tra ospedale e territorio. 
Sarà di fondamentale importanza il coinvolgimento attivo del PCAP che, come snodo organizzativo del Dipartimento di Cure Primarie, attiva il piano assistenziale per tutti i pazienti segnalati dall'Ospedale con modalità di Dimissione Protetta, compresi i pazienti portatori di lesioni cutanee complesse, coinvolgendo così i diversi professionisti tra cui l'infermiere Specialista Wound Care.

\section{Punti di forza}

Volontà della Direzione di perseguire l'implementazione di questo progetto; volontà della Direzione DATeR di valorizzare le competenze degli infermieri Specialisti in Wound Care e di dare rilievo al PDTA che coinvolge quasi interamente la componente infermieristica; volontà della componente infermieristica a tutti i livelli di fare rete; Presenza di una rete di referenti infermieristici di $1^{\circ}$ livello; Volontà di collaborazione tra infermieri con diverse competenze allo scopo di raggiungere la best practice; Utilizzo di sistemi informatici per la dimissione del paziente fragile con o senza lesioni cutanee.

\section{Punti di debolezza}

Difficoltà di una parte della componente clinica (MMG, specialisti, case manager) di coinvolgere/accettare l'infermiere specialista in Wound Care nella gestione dei pazienti con ferite difficili; necessità dei sistemi informatici di utilizzare la prescrizione medica per tracciare ciascun passaggio diagnostico e terapeutico; disomogeneità di conoscenze delle best practices da parte degli infermieri di $1^{\circ}$ livello, dei $\mathrm{MMG}$ e degli infermieri specialisti; mancanza di un sistema aziendale di monito- raggio delle lesioni cutanee e dell'appropriatezza di utilizzo dei dispositivi medici della categoria CDN M0404 Medicazioni Avanzate; disomogeneità della documentazione assistenziale-infermieristica utilizzata nelle degenze, negli ambulatori infermieristici e in assistenza domiciliare per tracciare e condividere la valutazione e il trattamento delle lesioni cutanee.

\section{Opportunità}

Valorizzare la figura degli infermieri Specialisti in Wound Care presenti in Azienda; dare risposta alla necessità di condividere conoscenze e competenze da parte degli infermieri che si occupano a tutti i livelli di lesioni cutanee; concentrarsi su un problema di salute del malato fino a ora diversificato per tipologia di lesioni; presenza di un consulente Specialista in Wound Care a cui far riferimento nel setting assistenziale; possibilità di eseguire consulenze riducendo il disagio del paziente per lo spostamento.

\section{Minacce}

Ritardo, da parte di MMG, specialisti e case manager dei diversi setting assistenziali, della richiesta di intervento su quelle lesioni che diventando croniche rendono più difficoltosi i tempi di guarigione; scarsa conoscenza del progetto e del ruolo del facilitatore della gestione delle lesioni cutanee; resistenza al cambiamento.

Tutto il progetto sarà accompagnato dalla formazione continua che ha come obiettivo proprio l'omogeneizzazione delle conoscenze ma anche la conoscenza fra gli attori della rete in quanto i corsi prevedono la presenza degli operatori di tutti i setting interessati (Tabella 1).

Tabella 1. Tempogramma di Gantt.

\begin{tabular}{|c|c|c|c|}
\hline Intervento & Azione & $\begin{array}{l}\text { Tempo di } \\
\text { attuazione }\end{array}$ & Responsabile \\
\hline N. 1 & Informazione e comunicazione del progetto & 1 settimana & Infermiere Specialista Wound Care \\
\hline N. 2 & Stesura del progetto & 4 mesi & $\begin{array}{l}\text { Infermiere Specialista Wound Care, DATeR Pianura Ovest, } \\
\text { Responsabile Assistenziale PDTA Ferite Difficili }\end{array}$ \\
\hline N. 3 & $\begin{array}{l}\text { Presentazione e condivisione con DATeR AUSL } \\
\text { Bologna }\end{array}$ & 1 mese & $\begin{array}{l}\text { DATeR Pianura Ovest, Responsabile Assistenziale PDTA Ferite } \\
\text { Difficili }\end{array}$ \\
\hline N. 4 & $\begin{array}{l}\text { Accordi con Coordinatori Area Degenza Ospedaliera, } \\
\text { Area Territoriale, Area Ambulatori distrettuali }\end{array}$ & 2 settimane & $\begin{array}{l}\text { DATeR Pianura Ovest, Responsabile Assistenziale PDTA Ferite } \\
\text { Difficili }\end{array}$ \\
\hline N. 5 & $\begin{array}{l}\text { Condivisione con gruppo infermieristico e Medici di } \\
\text { Medicina Generale }\end{array}$ & 1 mese & $\begin{array}{l}\text { DATeR Pianura Ovest, Responsabile Assistenziale PDTA Ferite } \\
\text { Difficili, Infermiere Specialista Wound Care }\end{array}$ \\
\hline N. 6 & $\begin{array}{l}\text { Avvio sperimentazione Specialista Wound Care, } \\
\text { Facilitatore gestione lesioni cutanee }\end{array}$ & 6 mesi & Infermiere Specialista Wound Care \\
\hline N. 7 & $\begin{array}{l}\text { Incontro e rivalutazione progetto con Coordinatori } \\
\text { Area Ospedaliera, Territoriale e di Distretto }\end{array}$ & 1 settimana & $\begin{array}{l}\text { DATeR Pianura Ovest, Responsabile Assistenziale PDTA Ferite } \\
\text { Difficili, Infermiere Specialista Wound Care }\end{array}$ \\
\hline N. 8 & $\begin{array}{l}\text { Presentazione dati alla Dirigenza Infermieristica } \\
\text { Aziendale }\end{array}$ & 1 mese & $\begin{array}{l}\text { DATeR Pianura Ovest, Responsabile Assistenziale PDTA Ferite } \\
\text { Difficili }\end{array}$ \\
\hline N. 9 & Avvio del progetto definitivo & 1 mese & Infermiere Specialista Wound Care \\
\hline
\end{tabular}




\section{Indicatori di processo e di esito}

\section{Numero di lesioni insorte nei pazienti dimessi in assenza di lesioni}

L'indicatore è rilevabile solamente tra pazienti dimessi dall'ospedale che non presentano lesioni da pressione, che hanno una scala di Braden uguale e/o inferiore a 18, e per i quali è stato attivato un percorso di dimissione protetta poiché per tali pazienti è previsto una tracciabilità tramite sistema gestionale informatizzato Garsia.

Verrà rilevata l'incidenza dei pazienti che hanno manifestato insorgenza delle lesioni da pressione sul totale dei pazienti dimessi in un determinato periodo di osservazione. Il dato d'incidenza sui periodi successivi osservati dovrà tendere verso un andamento in diminuzione.

\section{Supervisione e monitoraggio della gestione delle ferite cutanee complesse}

Numero consulenze effettuate per la gestione delle ferite cutanee.

Il personale coinvolto nella gestione delle lesioni cutanee dovrà fornire completa reportistica della tipologia delle lesioni, degli interventi effettuati e dei risultati ottenuti basandosi su indicatori standardizzati (ad es. WBS, classificazione EPUAP/NPUAP, classificazione TEXAS, ecc).

Prendendo ad esempio la classificazione EPUAP si possono considerare complesse le lesioni di $3^{\circ}$ e $4^{\circ}$ categoria che tendono all'intervento dell'infermiere Specialista Wound Care.

Non essendo standardizzabili i tempi e i modi di guarigione della lesione in quanto il Wound Healing è un processo influenzabile da fattori intrinseci ed estrinseci (ad esempio età, stato di salute, commorbilità, grado di mobilità, ecc.) l'indicatore dovrà rilevare il numero di richieste di intervento dell'infermiere Specialista per specifiche tipologie di categorie di lesioni, ad esempio $3^{\circ}$ e $4^{\circ}$ categoria.

Il risultato atteso, indicatore di esito, dovrà essere quello di dimostrare e confermare che vi sia una maggiore autonomia di gestione dei pazienti portatori di lesioni da pressione di $3^{\circ}$ e $4^{\circ}$ categoria da parte degli operatori e dei care givers attraverso la formazione continua ed il monitoraggio dei processi.

\section{Collegamento ospedale-territorio}

Numero di interventi effettuati in Ospedale $v s$ il Territorio.

Verrà attivato, tramite il PCAP, l'intervento dell'infermiere specialista per quei pazienti portatori di lesioni cutanee complesse prossimi alla dimissione a cui è stato attivato il percorso di dimissione protetta al domicilio. L'indicatore dovrà rilevare il numero di richieste di interventi dell'infermiere Specialista per il totale dei pazienti portatori di lesioni cutanee complesse, dimessi con modalità di dimissione protetta.

Il risultato atteso è che i pazienti portatori di lesioni complesse vengano dimessi con la valutazione dell'infermiere specialista il quale dovrà valutare lo stato del paziente, la tipologia di lesione, il trattamento in atto, a supporto della definizione del percorso assistenziale già strutturato e della continuità di cura.

\section{RISULTATI ATTESI}

L'implementazione di questo progetto permetterebbe di avvalersi di una figura professionale specialista che, esercitando la competenza specifica nella gestione del soggetto portatore di lesione cutanea, aiuterebbe i collaboratori nell'individuare tempestivamente i soggetti fragili e a rischio di insorgenza di lesioni cutanee e la loro gestione.

La collaborazione attiva tra l'infermiere Specialista facilitatore e colleghi delle Unità Assistenziali Ospedaliere e/o Territoriali dovrebbe poter trasferire un certo numero di prestazioni a livello territoriale determinando una diminuzione dei costi per le medicazioni, una riduzione del disagio per i pazienti non autosufficienti nonché un miglioramento della qualità di vita degli stessi attraverso il miglioramento dell'assistenza.

La tempestività di intervento su lesioni cutanee di difficile gestione porterebbe ad una riduzione dei tempi di degenza per quei pazienti ricoverati con diagnosi legate alle lesioni cutanee.

La metodologia del Coaching dovrebbe favorire una crescita motivazionale dei professionisti che in collaborazione con l'infermiere specialista continueranno a occuparsi di soggetti portatori di lesioni cutanee nei diversi Setting Assistenziali.

\section{DISCUSSIONE}

L'impatto sociale ed economico con l'implementazione di questo progetto, sarà sicuramente positivo, la sua realizzazione sicuramente di non facile attuazione poiché gli interessi per la fattibilità dovranno essere compresi ed elaborati a più livelli di responsabilità.

In una realtà assistenziale/organizzativa/professionale attuale, dove sempre più l'interesse va verso il risparmio e l'ottimizzazione delle risorse, sia economiche che umane, e dove la qualità dell'assistenza erogata è orientata verso le evidenze scientifiche ma soprattutto la qualità dell'assistenza percepita è fondamentale per la creazione di un'alleanza terapeutica e per il raggiungimento degli outcome di esito, la figura di un facilitatore nella gestione delle lesioni cutanee, con un approccio metodologico di Coaching, favorirà una tempestività nelle prestazioni da 
erogare superando i limiti dei tempi d'attesa, una possibilità di affiancamento, direttamente sul setting assistenziale dello Specialista Wound Care nei momenti di criticità legati all'assessment delle ferite.

Questo influirà positivamente sui costi relativi agli spostamenti dell'utente non autosufficiente portatore di lesione cutanea; sulle liste d'attesa degli ambulatori di $2^{\circ}$ livello del PDTA delle ferite difficili; sull'intercettazione dei pazienti fragili ed a rischio di insorgenza di lesioni da pressione favorendo le misure di prevenzione in maniera anticipatoria; sulla formazione on work direttamente sul campo operativo, attraverso il ragionamento clinico e pratico, migliorando le performance di competenza degli operatori coinvolti per il raggiungimento delle best practice.

Il coinvolgimento degli operatori, in quelle situazioni di difficile gestione, favorirà una crescita motivazionale al fine del raggiungimento degli obiettivi personali e professionali, attraverso l'affiancamento, la gratificazione e l'incitamento al miglioramento.

\section{CONCLUSIONI}

Attraverso questa modalità di gestione delle lesioni cutanee si offrirà all'infermiere specialista Wound Care la possibilità di rendere evidente la sua professionalità, la sua competenza specifica, di agire in maniera autonoma e di assumersi quella responsabilità professionale di cui tanto si parla ma che, ancora oggi, è limitata da antiche resistenze e da modelli organizzativi rigidi.

Il facilitatore specialista Wound Care sarà una figura dirimente a supporto della Dirigenza Assistenziale speri- mentandosi nella raccolta dati, nei progetti di ricerca innovativi e funzionali alla prevenzione, alla valutazione e al trattamento delle lesioni cutanee sia in aree territoriali che ospedaliere.

\section{BIBLIOGRAFIA}

1. Agenzia per Sanità e la ricerca di qualità 2011; NPUAP/EPUAP/PPPIA 2014. Available from: http://salute.regione.emilia-romagna.it/farmaci/dispositivi-medici

2. Piano della Performance Azienda AUSL di Bologna 20152017. Available from: https://www.ausl.bologna.it/asl-bologna/staff/so-form/sopf-trasparenza/piano-della-performance

3. Commissione Regionale Dispositivi Medici, Direzione Generale Sanità e Politiche Sociali e per l'Integrazione. Le medicazioni avanzate per il trattamento delle ferite acute e croniche - Servizio Assistenza Territoriale -Area Farmaco e Dispositivi Medici, 2016. Available from: https://salute.regione.emilia-romagna.it/documentazione/documenti-tecnici

4. Guida alla scelta della medicazione Commissione Aziendale dei Dispositivi Medici Segreteria c/o Farmacia Centralizzata Ospedale Maggiore Azienda USL di Bologna. Available from: http://www.saluter.it/ssr/aree-dellassistenza/assistenza-farmaceutica/dispositivi-medici

5. Commissione Regionale Dispositivi Medici Regione Emilia Romagna. Protocollo per il trattamento delle lesioni da pressione. 2015. Available from: http://salute.regione.emilia-romagna.it/farmaci/dispositivi-medici

6. Protocollo di trattamento delle lesioni vascolari dell'arto inferiore. Le medicazioni avanzate per il trattamento delle ferite acute e croniche. 2016. Available from: http://salute. regione.emilia-romagna.it/farmaci/Allegato5Protocollolesionivascolaridefinitivo.pdf/view 